\title{
LO SCUDO DI CANTO
}

Summary: The study examines one of the shield-descriptions of Valerius Flaccus' Argonautica. Its main aim is to demonstrate that Valeris Flaccus altered Canthus' story in accordance with his literary purposes. The poet depicts the shield of Canthus in the catalogue of the Argonauts mentioning that the hero had inherited this famous shield from his father, Abas, albeit according to the mythology he is not known to have any shield. The paper displays how many other Abases there were in Greek mythology and in Roman literature having a shield and it is argued that Valerius Flaccus was influenced by the coincidence of names and transformed the original story of Canthus (which can be read in Apollonius Rhodius) in order to imitate his literary models: Vergil, Ovid and the Iliad. Furthermore, the author rewrites the story of Canthus so that the Argonaut can be paralleled with Patroclus. Consequently, Canthus must be an important person of the epic which is highlighted by Valerius Flaccus in several ways and his shield has to have a literary function.

Key words: Valerius Flaccus, Argonautica, Canthus, Abas, Patroclus, Iliad, shield description, ekphrasis

Nel primo libro degli Argonautica Valerio Flacco elenca gli Argonauti che partono verso la Colchide per acquisire il Vello d'oro. Uno di loro è Canto il cui scudo sarà descritto nella seguente ecfrasi:

insurgit transtris et remo Nerea versat

Canthus, in Aeaeo volvet quem barbara cuspis

pulvere; at interea clari decus adiacet orbis

quem genitor gestabat Abas (secat aurea fluctu

tegmina Chalcidicas fugiens Euripus harenas

celsaque semiferum contorquens frena luporum

surgis ab ostrifero medius, Neptune, Geraesto).

Arg. 1. 450-456

L'ecfrasi stessa comincia soltanto nella linea 453 con il verbo secat ma il poeta, prima della descrizione dello scudo, prelude al fato di Canto, coiè che lui morirà in Aia (dunque nella Colchide), nella guerra contro il re Perse. Valerio Flacco, quindi, 
trasforma la storia di Canto che viene descritta anche nelle Argonautiche di Apollonio Rodio, ma nell'epopea greca Canto è ucciso dal Cafauro perché gli rapisce le pecore (cf. Ap. Rhod. 4. 1485-1501).

Nel mio studio vorrei esporre quali sono i motivi per cui Valerio Flacco trasforma la storia. L'occorrenza del nome Abante (genitore di Canto) crea l'occasione di imitare altre opere letterarie. Imitando anche l'Iliade di Omero, Valerio Flacco rende Canto un personaggio importante dopo la morte del quale si sviluppa una battaglia per il suo corpo e le sue armi come nel caso di Patroclo. Quando muore, Patroclo porta le armi di Achille perciò anche la descrizione dello scudo di Canto può essere paragonato allo scudo dell'eroe greco e ha la stessa funzione letteraria - anche se Patroclo porta le prime armi di Achille e non lo scudo famoso.

Prima della descrizione dello scudo Valerio Flacco scrive che Canto l'ha ereditato da suo padre, Abante, re dell'Eubea, figlio di Nettuno e della ninfa Aretusa (cf. Hyg. Fab. 157). Anche se secondo la mitologia questo Abante non aveva nessun scudo la mitologia greca e la letteratura romana invece conoscono alcune persone chiamate Abanti che possiedono uno scudo il quale è stato ereditato o conquistato da qualcuno. Il più famoso tra quelli è il figlio di Ipermnestra, l'unica delle Danaidi che salva la vita del marito. Quando Abante avvisa suo padre, Linceo (il marito salvato) della morte di Danao, Linceo per la felicità gli regala il suo scudo che antecedentemente è stato offerto ad Era (Hyg. Fab. 170; 273).

Anche nell'Eneide di Virgilio si trova un episodio dove Enea offre ad Apollo ad Azio uno scudo che ha preso da un milite chiamato Abante:

aere cavo clipeum, magni gestamen Abantis,

postibus adversis figo et rem carmine signo:

Aeneas haec de Danais victoribus arma;

linquere tum portus iubeo et considere transtris.

Aen. 3. 286-289

I commentatori di Virgilio spiegano diversamente chi poteva essere questo Abante. Secondo Servio, Abante è uno degli Achei da cui i militi di Enea prendono armi dopo averli uccisi insieme ad Androgeo (Verg. Aen. 2. 370-401). Secondo il commentario di Servio Danielino egli è il figlio di Linceo ma questo sarebbe un anacronismo poiché la storia delle Danaidi precede la guerra di Troia con più generazione. C'è anche la possibilità che si tratta di un postero di Linceo, Abante che ha ereditato lo scudo. ${ }^{1}$ Il figlio di Linceo ebbe veramente un bisnipote chiamato Abante (Apd. 2. 2. 2) ${ }^{2}$ ma lui fu il padre dell'Argonauta, Idmone (Ap. Rhod. 2. 817-818), ${ }^{3}$ dunque visse generazioni prima della guerra di Troia, coiè si tratterebbe di nuovo di un'anacronismo se Enea gli avesse preso lo scudo. Probabilmente nell'episodio di Virgilio non è importante il personaggio di Abante (anche se il sintagma magni Abantis dovrebbe indicare

\footnotetext{
${ }^{1}$ MiLler, J. F.: The Shield of Argive Abas at Aeneid 3. 286. CQ 43 (1993) 445-450, 446.

${ }^{2}$ Abante aveva una figlia, Idomene, lei aveva un figlio, Melampo, il cui figlio, Abante era il padre di Idmone. Sebbene altronde si legge che il padre di Idomene era Perete (Apd. 1. 9. 11).

${ }^{3}$ Secondo un'altra variazione il padre di Idmone era Apollo.
} 
una persona famosa ${ }^{4}$ ) ma con il fatto che Enea offre arme prese dal nemico Virgilio vuole alludere all'offerta di Agosto fatta dopo la vittoria della battaglia di Azio.

Ovidio imita la scena di Virgilio:

Panthoides Euphorbus eram, cui pectore quondam

haesit in adverso gravis hasta minoris Atridae; cognovi clipeum, laevae gestamina nostrae, nuper Abanteis templo Iunonis in Argis!

Met. 15. 161-164

In questo episodio Pitagora - che nella sua vita precedente era Euforbo - vede uno scudo che gli ha preso Menelao. Lo scudo adesso è nel tempio di Giunone nella città di Argo, dunque in quel tempio dove era stato offerto lo scudo di Linceo. Ovidio pone le parole clipeum e gestamina ${ }^{5}$ allo stesso luogo della linea 163 dove si trovano nella linea 286 dell'Eneide. Ovidio inoltre invertisce la scena di Virgilio: Enea di origine di Troia offre uno scudo preso da un greco mentre qui si tratta di uno scudo che un greco ha preso da un troiano. ${ }^{6}$

La descrizione dello scudo di Valerio Flacco ci fa venire in mente la storia di Linceo e le due citazioni dei poeti antenati. Sopratutto perché anche qui si tratta di uno scudo ereditato da uno chiamato Abante ma anche perché ci si trovano parole simili a quelle usate da Virgilio ed Ovidio. Benché Valerio Flacco non usa le parole clipeum e gestamina, ma la parola gestabat (Arg. 1.453) derivata dalla stessa radice come la forma gestamina e la forma della parola tanstris (Arg. 1.450) che si trova anche nel tratto di Virgilio.

La descrizione dello scudo degli Argonautica allude anche alla descrizione dello scudo di Enea poiché tutti e due contengono il verbo seco (che può significare: varcare il mare) e l'idea dell'oro e della fluttuazione (Arg. 1. 453; Verg. Aen. 8. 671; 8. 677). L'immagine dello stretto che varca la terraferma (Arg. 1. 453-454) è stata probabilmente ispirata dalla descrizione virgiliana dei delfini varcanti il mare. ${ }^{7}$ La funzione della descrizione dell'Eneide è l'elogio dell'imperatore e anche le immagini collegate al mare dello scudo di Canto possono farci venire in mente lo scopo politicale degli Argonautica: la celebrazione di Vespasiano per i suoi successi marini. La descrizione dello scudo di Canto però non ha una prefigurazione letteraria poiché non conosciamo le decorazioni degli scudi menzionati da Virgilio e Ovidio, poi non sappiamo neanche della decorazione dello scudo di Linceo.

In base di tutto questo, il nome Abante dà occasione a Valerio Flacco di imitare altre opere letterarie. Imitando anche l'Iliade, trasforma la storia di Canto e lo rende un personaggio paragonabile a Patroclo. ${ }^{8}$

\footnotetext{
${ }^{4}$ Miller (n. 1) 445.

${ }^{5}$ Virgilio è il primo a usare la parola gestamen nel senso di armatura (OLD ad loc.) e Anche Valerio Flacco l'usera così (cf. Arg. 3. 344).

${ }^{6}$ Miller, J. F.: The Memories of Ovid's Pythagoras. Mnemosyne 47 (1994) 437-487, 480.

${ }^{7}$ KLeywegt, A. J.: Praecursoria Valeriana III. Mnemosyne 41 (1988) 355-372, 363.

${ }^{8}$ WiJsman, H. J. W.: Gesander alter Mezentius. Valerius Flaccus 6. 279-385. Mnemosyne 51 (2000) $58-70,64$.
} 
Negli Argonautica Telamone difende il cadavere di Canto come un leone i suoi cuccioli e lo copre con il suo scudo di sette strati:

at vero ingentem Telamon procul extulit orbem

exanimem te, Canthe, tegens. ceu saeptus in arto

dat catulos post terga leo, sic comminus astat

Aeacides gressumque tenet contraque ruentem

septeno validam circumfert tegmine molem.

Arg. 6. 345-349

Nell'Iliade Aiace, figlio di Telamone difende il corpo di Patroclo con il suo scudo che è similmente di sette strati e troviamo anche la similitudine con il leone (cf. Hom. Il. 17. 132-137). Il cadavere di Patroclo è teso come una pelle di toro (Hom. Il. 17. 389-95) e questo succede anche a Canto: ${ }^{9}$

ut bovis exuvias multo qui frangit olivo

dat famulis, tendunt illi tractuque vicissim

taurea terga domant, pingui fluit unguine tellus.

talis utrimque labos raptataque limite in arto

membra viri miseranda meant. hi tendere contra,

hi contra alternaeque virum non cedere dextrae.

Arg. 6. 358-363

Se si sviluppa una battaglia per il corpo di Canto, lui deve essere un personaggio importante e questo sarà veramente sottolineato più volte da Valerio Flacco. Canto è menzionato prima volta da Giasone come un eroe merito a cercare il Vello d'oro insieme a Telamone e Ida: ${ }^{10}$

ductor ait: 'non degeneres, ut reris, Acaste, venimus ad questus: socium te iungere coeptis

est animus neque enim Telamon aut Canthus et Idas

Tyndareusque puer mihi vellere dignior Helles. Arg. 1. 164-167

Canto si esibisce anche nel terzo libro dell'epopea: combatte contro i militi di Cizico (re dei Dolioni) dopo che gli Argonauti sono costretti, a causa di una tempesta di notte, a ritornare ai Dolioni e non sono riconosciuti da loro. Canto alla fine non uccide nessuno poiché Cidro vulnerato da lui sarà colpito da Polluce:

tum Castor Ityn, qua caerulus ambit

balteus et gemini committunt ora dracones,

frater Hagen Thapsumque securigerumque Nealcen

transigit et Canthi pallentem vulnere Cydrum.

Arg. 3. 189-192

Canto così acquisisce un po’ di aristia senza uccidendo qualcuno fra i Dolioni, amici degli Argonauti. ${ }^{11}$ 370,368

${ }^{9}$ BARNES, W. R.: The Trojan War in Valerius Flaccus' Argonautica. Hermes 109 (1981) 360-

${ }^{10}$ Anche Ida difenderà il cadavere di Canto (Arg. 342)

${ }^{11}$ DINTER, M. T.: Epic from Epigram: The Poetics of Valerius Flaccus' Argonautica. AJPh 130 (2009) 533-566, 550 . 
La ripetizione del nome di Canto nella scena che racconta la sua morte mette anche in risalto la sua importanza. Durante la descrizione della battaglia per il corpo di Canto (Arg. 6. 364-372) si esibisce il nome tre volte: una volta nella linea 364 e due volte nella linea 368. Questa ripetizione esprime forse lo squasso del cadavere qua e là. ${ }^{12}$

Il nome di Canto apparisce una volta in più dopo la sua morte. Giasone rievoca a Medea che Canto sia morto per la Colchide da una punta di lancia di estero:

an iacet externa quod nunc mihi cuspide Canthus

quodque meus vestris cecidit pro moenibus Iphis

aut Scythiae tanta inde manus?

Arg. 7. 422-424

L'espressione externa cuspide si riferisce all'espressione barbara cuspis che si trova nelle righe precedenti dell'ecfrasi. Le due espressioni così danno una cornice alla storia di Canto che sottolinea di nuovo la sua importanza. ${ }^{13}$

Giasone menziona a Medea anche Ifi (Arg. 7. 423) che è morto lo stesso per la Colchide e la cui morte è predetta da Valerio Flacco (Arg. 1.441) similmente a quella di Canto (Arg. 1. 451). Un parallelo ulteriore tra di loro è che sia Ifi (Arg. 1. 443) che Canto (Arg. 6.317) sono stati pianti dalla nave Argo. Tra le variazioni della storia gli Argonautica di Valerio Flacco sono gli unici ad introdurre Ifi. Nell'epopea si esibisce due volte: nel catalogo dove la sua morte è predetta e quando Giasone lo menziona a Medea. Dunque la battaglia in cui muore non è raccontata. Questi paragoni con Canto e la mancanza della descrizione della sua morte servono a mettere in risalto l'importanza della morte di Canto. Gesandro, l'assassino di Canto invece si esibisce soltanto nell'episodio che descrive la morte dell'Argonauta e non è elencato nemmeno nel catalogo dei militi di Perse. Tutto questo può alludere al suo ruolo nefando.

Poiché dopo la morte di Canto la battaglia non è soltanto per il suo corpo ma anche per le sue armi, anche queste armi devono essere importanti. Nel primo libro degli Argonautica anche Valerio Flacco parla di uno scudo famoso (clari decus orbis). L'attributo clari può significare anche la preziosità dello scudo. Quest'è una spiegazione ulteriore della battaglia per lo scudo. Sebbene Valerio Flacco non menziona di nuovo le armi nel sesto libro dell'epopea, probabilmente la causa di questo è la « composizione economica » usata dal poeta. ${ }^{14} \mathrm{Ci}$ sono invece alcune parole che possono essere interpretate come riferimenti allo scudo. Valerio Flacco indica lo scudo di Telamone con la parola tegimen (Arg. 6.349) che è stata usata anche nell'ecfrasi con il significato di scudo. Menziona poi la patria di Canto, Eubea (Arg. 6.321) che è stata rappresentata sullo scudo.

Come abbiamo visto prima, Canto può essere paragonato a Patroclo nel senso che alla sua morte porta le armi di Achille per questo anche lo scudo di Canto può essere paragonato a quello dell'eroe greco e così anche la descrizione dello scudo di Canto deve avere una funzione letteraria. Il ruolo della descrizione dello scudo di Achille in parte è di rappresentare il mondo del periodo dell'Iliade mentre le immagini

\footnotetext{
${ }^{12}$ MiLleR: The Memories (n. 6) 464.

13 Zissos, A.: Valerius Flaccus' Argonautica: A Commentary. Book I. Oxford 2005, 289.

${ }^{14}$ WiJSMAN (n. 8) 68.
} 
sullo scudo di Canto (lo stretto Euripe e Nettuno) rievocano il mondo degli Argonauti: il mare. L'idea della rappresentazione del mondo ci può venire in mente anche per la parola orbis usata in senso di scudo poiché questa parola significa anche mondo - principalmente nell'espressione orbis terrarum.

Sullo scudo si vede Euripe, lo stretto di mare che separa Eubea da Beozia, la terraferma greca accanto alla città di Calchi. Questo è stato rappresentato sullo scudo indiscutibilmente per alludere all'origine di Canto siccome suo padre, Abante era il re di Eubea e nell'antichità gli scudi avevano molte volte la funzione di visualizzare l'origine geografica del proprietario. ${ }^{15}$

Secondo la descrizione Euripe è rappresentato su uno scudo aureo (aurea tegmina). L'oro segnala da una parte la preziosità dello scudo e spiega meglio anche il motivo della battaglia, dall'altre parte significa anche la sabbia aurea di Calchi che è il contrasto del polvere della Colchide. ${ }^{16}$ Il paesaggio piacevole rappresentato sullo scudo di Canto è dunque il contrario del paesaggio barbaro dove l'Argonauta morirà ${ }^{17} \mathrm{La}$ bellezza del paesaggio dipinto proietta così il pathos della morte di Canto. ${ }^{18}$ Il polvere di Aia può essere contraposto anche al mare perché Canto lo domina remando sulla nave Argo mentre gli è pericolosa la terraferma. Sull'immagine dello scudo c'è Nettuno a dominare il mare, Euripe invece fugge (fugiens) la terraferma, la sabbia di Calchi. Dunque le immagini dello scudo possono esprimere che la terraferma ossia la Colchide sarà pericolosa per Canto. ${ }^{19}$

Sullo scudo c'è Nettuno emergendo dal mare su un carro trainato da animali metà lupi metà pesci. ${ }^{20}$ Nettuno c'è sullo scudo perché egli è un antecedente di Canto: era il padre di Abante, padre di Canto, mentre la madre di Abante era la ninfa Aretusa. Le immagini dello scudo alludono così all'origine familiaria di Canto che è una funzione ulteriore degli scudi antichi. ${ }^{21}$ Prima dell'ecfrasi Valerio Flacco indica il mare con la parola Nerea e anche questo può essere interpretato come un'allusione all'origine di Canto poiché Nereo era il padre di Aretusa.

Il verbo secat con cui inizia l'ecfrasi e il quale si riferisce allo stretto di Euripe si trova nel mezzo di sette righe che si trattono di Canto e le divide in due parti tra quali ci sono paralleli e contrari. La prima parte è più vivace mentre la seconda è più tranquilla sopratutto perché alcune delle azioni rappresentate sullo scudo sono espresse da participi e non da verbi. Il movimento di Canto e di Nettuno invece è espresso quasi dagli stessi verbi insurgit e surgis. Un altro paragone tra di loro che tutti e due sono su un " veicolo d'acqua »: Canto sulla nave Argo, Nettuno sul suo

${ }^{15}$ CHASE, G. H.: The shield devices of the Greeks. HSCPh 13 (1902) 61-127, 92.

${ }^{16}$ AsQuITH, H. C. A.: Silver epic catalogues. Durham theses, Durham University, 2001, 129. Accessibile su Durham E-Theses Online: http://etheses.dur.ac.uk/4368/.

${ }^{17}$ DiNTER (n. 9) 548.

${ }_{18}^{18}$ ASQUiTH (n. 14) 129.

${ }^{19}$ È una congruenza interessante ma probabilmente non intenzionale che i nomi originali delle città di Calchi (Chalkis) e della Colchide (Colchis) sono molto simili.

${ }^{20}$ Secondo la mitologia non esistono tali animali e quelli dovrebbero essere cavalli marini cioè si tratta probabilmente di una corruttela (KLEYWEGT [n. 7] 365-367). Ossia ci sono i lupi perché lo scudo abbia una funzione ulteriore generale: spaventare il nemico.

${ }^{21}$ CHASE (n. 13) 92. 
carro. Il mortale Canto può dunque essere rassomigliato alla divinità immortale ma lui è anche il suo contrario poiché muore. Forse non è nemmeno a caso che l'arma che lo uccide è una lancia (cuspis) poiché la parola cuspis può denotare il tridente di Nettuno - anche Valerio Flacco l'usa in quel senso (Arg. 2. 618). Secondo la descrizione poi Nettuno è rappresentato su un carro mentre anche il cadavere di Canto sarà posto su un carro (Arg. 6.370).

In base di tutto questo quasi ogni parola della descrizione ha il proprio ruolo. La descrizione è dunque un'ecfrasi ben elaborata ed ha veramente una funzione letteraria come abbiamo aspettato.

Gábor Hamvas 